\title{
The Significance of High-throughput Genome Sequencing for the Diagnosis of Neonatal and infant Septicemia
}

\author{
N Zou, L Yang, J Zhu, MM Zhang and ZJ Liu* \\ Department of Pediatrics, The Second Affiliated Hospital of Dalian Medical University, China
}

*Corresponding author: ZJ Liu, Department of Pediatrics, The Second Affiliated Hospital of Dalian Medical University, China

\begin{tabular}{lll}
\hline ARTICLE INFO & & ABSTRACT \\
\cline { 1 - 1 } $\begin{array}{ll}\text { Received: } \\
\text { Published: July 17, } 2019\end{array}$ & & $\begin{array}{l}\text { Citation: N Zou, L Yang, J Zhu, MM Zhang, ZJ Liu. The Significance of High-throughput } \\
\text { Genome Sequencing for the Diagnosis of Neonatal and infant Septicemia. Biomed J Sci \& } \\
\text { Tech Res 20(1)-2019. BJSTR. MS.ID.003389. }\end{array}$ \\
\hline
\end{tabular}

\section{Introduction}

Neonatal septicemia lacks typical manifestations. Since the neonatal and infant immune system is immature and the immune function is poor, infection is very likely to occur. Infection will hardly localized and lead to extensive inflammatory response across the body. Thus, the condition progresses rapidly and become severe [1]. Currently, blood culture remains to be the golden standard for the diagnosis of septicemia. However, the positive rate of culture of newborns with septicemia is low, the culture time is long, and it is hard to select specific antibiotics at the early stage, making it very difficult to control the disease. High-throughput genome sequencing technology involves in the direct acquisition of nucleotide sequence information about the pathogens screened out by using the next generation sequencing (NGS), as well as the comparative analysis of the sequences by biological information method. Based on the characteristics of no bias and randomness, such technology can detect the genome of a known pathogen and thus provide assistance for early clinical diagnosis [2-4]. We reported the application of high-throughput genome sequencing technology in the diagnosis of neonatal and infant septicemia and provide evidence for its early diagnosis in clinical practice.

\section{Case 1}

A premature with 34 weeks gestational age was admitted into our NICU in May 2018. The boy was a small for gestational age infant with a birth weight of $1580 \mathrm{~g}$. The patient's mother had a history of severe preeclampsia and gestational diabetes mellitus. The Apargr scores were 5, 8 and 9 at $1 \mathrm{~min}, 5 \mathrm{~min}$ and $10 \mathrm{~min}$ after birth respectively. T:36, $7^{\circ} \mathrm{C}, \mathrm{P}: 148 / \mathrm{min}, \mathrm{R}: 44 / \mathrm{min}, \mathrm{Bp}: 73 / 51$ mmHg; Arterial blood gas analysis: PH:7.19, PCO2:37mmHg, P02:67mmHg, HCO3:-15.7mmol/L, BE:-10mmol/L. Brain MRI suggested cerebral injury. Nutritional therapy (for brain cells) and other symptomatic treatment were given. However, the discharge of the initial meconium was delayed. On the fifth day of birth, the baby was weak suction with less activity and abdominal distention. T:36, $9^{\circ} \mathrm{C}, \mathrm{P}: 168 / \mathrm{min}, \mathrm{R}: 51 / \mathrm{min}, \mathrm{Bp}: 79 / 52 \mathrm{mmHg}$. Blood routine test: WBC: $18.01 \times 109, \mathrm{~N}: 61.3 \%$, L: $21.7 \%$, HGB: $158 \mathrm{~g} / \mathrm{L}$, PLT: $100 \times 109$; CRP: $85.39 \mathrm{mg} / \mathrm{L}$. Blood culture was conducted and high-throughput genome sequencing was performed simultaneuously. The diagnosis was considered as intestinal septicemia and Meropenem was used for anti-infection, and fasting, gastrointestinal decompression, intravenous nutrition and other measures were taken. Klebsiella aerogenes was detected in $48 \mathrm{~h}$ by high-throughput genome sequencing (sequence number: 12; coverage: 0.0083\%), supporting the clinical diagnosis. The baby has been in remission. The blood culture on the fifth day reported Klebsiella aerogenes infection, which confirms the correctness of high-throughput genome sequencing.

\section{Case 2}

A three-month-old boy was admitted into our ward of the Second Hospital of Dalian Medical University for fever more than 10 days in Augus 2018. The body temperature was $39.2^{\circ} \mathrm{C}$ with no chill, convulsion, vomiting or diarrhea, and had 10 days history of Antibiotic therapy empirically for urinary system infection. T:39 ${ }^{\circ} \mathrm{C}, \mathrm{P}: 140 / \mathrm{min}, \mathrm{R}: 38 / \mathrm{min}, \mathrm{Bp}: 82 / 54 \mathrm{mmHg}$; Urine routine test: WBC: 52.35 /HP; Blood routine test: WBC:10.44×109, N: 63.9\%, L:23.7\%, HGB:100g/L, PLT: 351×109; CRP: 61.48mg/L. Blood culture and urine culture were performed, and high-throughput genome sequencing for pathogenic organisms was also performed simultaneuously. The cefoperazone was administrated empirically, 
and other symptomatic and supportive treatments were also given But the baby condition has not improved. The staphylococcus hominis was detected by genome sequencing (sequence number: 81; coverage: $0.6412 \%$ ) in 48 hours and linezolid replacded cefoperazone immediately. The blood culture confirmed the staphylococcus hominis which was broad spectrum drug-resistant bacteria sensitive to linezolid on the fifth day of blood collection. The baby symptoms eased rapidly and was found bladder-right ureter reflex by retrograde urography.

\section{Discussion}

The traditional blood culture technology requires a long period with a high false negative rate and a high contamination rate. In contrast, high-throughput genome sequencing technology can directly determine the nucleotide sequences of microorganisms in specimen and identify molecules [5-7] by comparing with the nucleotide sequences of standard microorganisms in databases. High-throughput genome sequencing technology saves the trouble of pre-test culture and can directly non-specifically determine all the nucleotide fragments without prior selection of the testing range (unbiasedness), thereby minimizing the loss detecting rate and detecting the host gene to assist the diagnosis $[8,9]$. In addition, the high-throughput genome sequencing can directly realize strain typing so as to obtain information about molecular serotype classification, resistance gene spectrum and so on, analyze the evolution history, infection source and infection route of the strain and contribute to the prevention, diagnosis and treatment of diseases [10]. It can be seen from the two cases that highthroughput genome sequencing technology requires a short time to detect pathogenic bacteria, and its testing accuracy is high, which is confirmed by blood culture. Such this technology provides a new approach to the early diagnosis of neonatal and infant septicemia.

\section{Conflict of Interest}

The authors declare that they have no conflicts of interest.

\section{References}

1. Thukral A, Sankar MJ (2017) Probiotics for prevention of suspected sepsis in low birthweight infants. J Acta Paediatrica 106(4): 681.

2. Keiju SK, Kontula, Kirsi Skogberg, Jukka Ollgren, Asko Järvinen, et al. (2018) The outcome and timing of death of 17,767 nosocomial bloodstream infections in acute care hospitals in Finland during 19992014. J European Journal of Clinical Microbiology \& Infectious Diseases 37(5): 945-952.

3. Lamping F, Jack T, Rübsamen N (2018) Development and validation of a diagnostic model for early differentiation of sepsis and non-infectious SIRS in critically ill children - a data-driven approach using machinelearning algorithms. J Bmc Pediatrics 18(1): 112.

4. Roberts JL, Patel RM (2017) Antibiotic utilization in very low birth weight infants without sepsis or necrotizing enterocolitis is associated with multiple adverse outcomes. J Evid Based Med 22(5): 187.

5. Ye M, Wei W, Yang Z (2016) Rapid diagnosis of Propionibacterium acnes infection in patient with hyperpyrexia after hematopoietic stem cell transplantation by next-generation sequencing: a case report. J BMC Infect Dis 16: 5 .

6. Yao M, Zhou J, Zhu Y (2016) Detection of Listeria monocytogenes in CSF from three patients with meningoencephalitis by next-generation sequencing. J Clin Neurol 12(4): 446-451.

7. Brown JR, Bharucha T, Breuer J (2018) Encephalitis diagnosis using metagenomics: application of next generation sequencing for undiagnosed cases. J Infect 76(3): 225-240.

8. Grumaz S, Stevens P, Grumaz C, Decker SO, Weigand MA, et al. (2016) Nextgeneration sequencing diagnostics of bacteremia in septic patients. J Genome Med 8(1): 73.

9. Segata N, Ballarini A, Jousson O (2013) Genome sequence of pseudomonas aeruginosa PA45 a highly vindent strain isolated from a patient with bloodstream infection. J Genome Announc 1(3): e00289-13.

10. Tong YG, Shi WF, Liu D (2015) Genetic diversity and evolutionary dynamics of Ebola virus in Sierra Leone. J Nature 524(7563): 93-96.

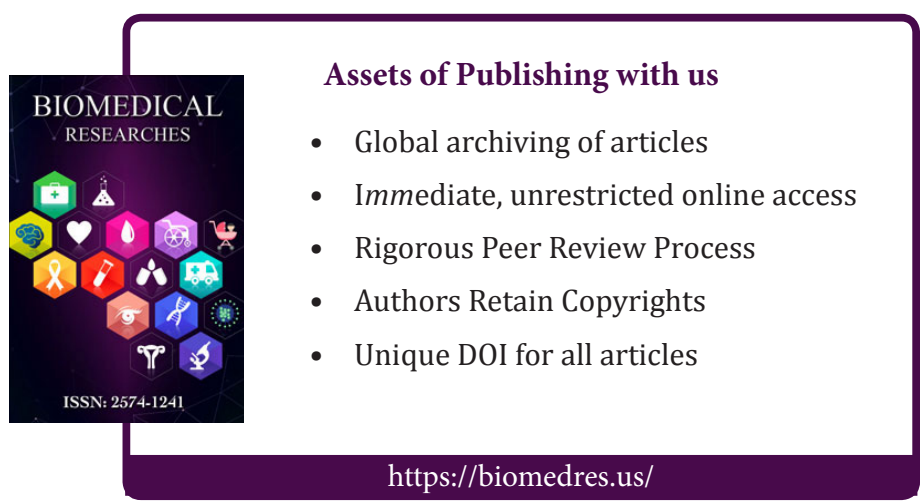

